\title{
El aprendizaje-servicio (APS) como práctica expansiva y transformadora
}

\author{
Azucena Ochoa Cervantes; Luis Manuel Pérez Galván; José Juan Salinas*
}

Resumen. La metodología de proyectos de Aprendizaje-Servicio en México, específicamente en el Estado de Querétaro ha sido impulsada recientemente. Como parte de las actividades de mejora de la convivencia del Observatorio de la Convivencia Escolar se desarrolla un programa piloto en 7 escuelas de nivel secundario de la ciudad de Querétaro con el objetivo de analizar el impacto de la metodología de proyectos de APS en la convivencia escolar, así como de valorar los resultados, para que, en el siguiente ciclo escolar se extienda a otras instituciones. Para alcanzar el objetivo, se realiza un estudio exploratorio de casos, la población es de 400 estudiantes, con el acompañamiento de 53 docentes. Los instrumentos de recolección de información son: cuestionario de participación infantil, escala de convivencia, observación estructurada. Dado que es un proyecto en curso, lo que se presenta en este trabajo son los avances acerca del proceso de la puesta en marcha de la metodología en las escuelas participantes. Como resultados parciales se destaca la modificación de las prácticas pedagógicas de los docentes, los cuales han adoptado en sus actividades escolares relaciones democráticas horizontales con los alumnos. Así mismo, se destaca el papel del alumnado como protagonista principal del proceso de APS al llevar a cabo el diagnóstico y la elección de la temática a intervenir, mostrando que esta estrategia permite mirar la parte social de la pedagogía pues lo que se promueve va más allá de los contenidos curriculares.

Palabras clave: Proyecto aprendizaje-servicio; convivencia escolar; educación secundaria.

\section{A APRENDIZAGEM EM SERVIÇO (APS) COMO UMA PRÁTICA EXPANSIVA E TRANSFORMADORA}

Resumo. A metodologia de projetos de Aprendizagem em Serviço no México, especificamente no Estado de Querétaro, recentemente ganhou novo impulso. Como parte das atividades de melhorias de convivência do Observatório da Convivência Escolar, está sendo desenvolvido um programa piloto em sete escolas secundárias da cidade de Querétaro, com o objetivo de analisar o impacto da metodologia de projetos de APS na convivência escolar, bem como de avaliar os resultados, de modo que, no próximo ano letivo, seja expandido a outras instituições. Para atingir o objetivo, é realizado um estudo exploratório de casos, onde a população é de 400 alunos, com o acompanhamento de 53 professores. Os instrumentos para a coleta de informações são: questionário de participação infantil, escala

* Observatorio de la Convivencia Escolar. Maestría en Educación para la Ciudadanía. Universidad Autónoma de Querétaro (UAQ), México. 
de convivência e observação estruturada. Dado que este é um projeto em andamento, o que é apresentado neste artigo são os avanços sobre o processo de implementação da metodologia nas escolas participantes. Como resultados parciais, é destacada a mudança das práticas pedagógicas dos professores, que adotaram as relações democráticas horizontais com os alunos em suas atividades escolares. Da mesma forma, ressalta-se o papel dos alunos como principal protagonista do processo de APS na realização do diagnóstico e na escolha do assunto para intervir, mostrando que esta estratégia permite observar a parte social da pedagogia, já que o que está sendo promovido vai além dos conteúdos curriculares.

Palavras-chave: Projeto de aprendizagem em serviço; convivência escolar; educação secundária.

LEARNING-SERVICE (APS) AS AN EXPANSIVE AND TRANSFORMATIVE PRACTICE Abstract. Learning-Service project methodology in Mexico specifically in Queretaro, has been improving. As part of the cooperation activities developed by the School Cooperation Observatory, a pilot project has been developed to be tested in 7 secondary schools in Queretaro City to analyze APS Project methodology's impact on school cooperation; Its results are an important part in order to implement the same system in other schools. To reach this goal, it is necessary to make an exploratory case study with 400 students and 53 supervisors as the sample. Data gathering tools are: Child participation surveys, cooperation ratio, and structured observation. This work reviews only the active school results of this methodology because it is an ongoing project.

As a partial result, we can observe a difference in teacher's pedagogical activities, whose had adopted democratical horizontal activities with their students, also we can highlight student participation as the main exponent in the APS project because they lead and choose the subject showing that this strategy allows us to see the social part of pedagogy because it goes beyond curricular contents.

Keywords: Learning-service project; school cooperation; secondary education.

\section{INTRODUCCIÓN}

Las realidades que como profesionales de la educación enfrentamos en relación con las formas de convivencia en las escuelas nos obliga a buscar alternativas de acción que nos permitan, desde la investigación, dar respuesta a los diversos retos que se viven hoy en las escuelas. Como lo hemos presentado en trabajos anteriores, consideramos que la convivencia en la escuela tiene dos dimensiones: una individual y otra institucional, es decir, que las formas en cómo nos relacionamos en el contexto escolar no sólo están determinadas por las características individuales de los miembros de la comunidad, sino que estas relaciones se ven condicionadas por las prácticas y políticas que se establezcan en una institución (Ochoa y Diez-Martínez, 2012). 
En este sentido cada escuela irá "enseñando" una forma particular de convivir a partir de la puesta en marcha de acciones cotidianas educativamente intencionadas - o no- que se dan en ese lugar, por lo que consideramos la convivencia escolar como un ámbito privilegiado para construir relaciones respetuosas y, al mismo tiempo, contribuir a la formación de la ciudadanía.

Sin embargo, observamos contradicciones de la institución (escuela) que tiene como uno de sus fines "Infundir el conocimiento y la práctica de la democracia como la forma de gobierno y convivencia que permite a todos participar en la toma de decisiones al mejoramiento de la sociedad" (Ley General de Educación, inciso V), puesto que de acuerdo a Bolívar (2016), la educación democrática tiene dos sentidos: educar para la democracia y, el ejercicio activo de la democracia, entonces, ¿cómo educar para la democracia y el ejercicio de la misma en una institución jerárquica?, ¿cómo educar para la democracia y el ejercicio de la misma en una institución en donde sus prácticas son autoritarias?, ¿cómo educar a las niñas y niños para la democracia y el ejercicio de la misma en una institución con una visión adultocéntrica?

A partir de lo anterior es que consideramos pertinente la mirada de la pedagogía social pues es necesario reflexionar sobre cuál es la misión de la educación, en este caso la "escolar", reflexionar en torno a sus políticas y sus prácticas y cuál es el resultado de éstas en la formación del individuo. Apostamos por una escuela que eduque para la democracia, entendiendo ésta como una forma de vida y práctica cotidiana en donde los miembros de una comunidad no sólo tienen el derecho sino el deber de participar en asuntos que son de su incumbencia.

De acuerdo a la UNESCO (1993), educar para la democracia implica empoderar a las y los estudiantes para participar activamente en todos los ámbitos de la vida social y política. Es por lo anterior que consideramos la participación como el eje transversal que permite la mejora de la convivencia y la formación de la ciudadanía activa.

El Menú de indicadores y Sistema de monitoreo del derecho a la participación de niños, niñas y adolescentes, proporciona una definición operativa de participación infantil al considerarla como proceso participativo en la medida que se hace efectivo el derecho de los niños, niñas y adolescentes a ser informados, emitir opinión, ser escuchados e incidir en las decisiones que se toman respecto de temas que son de su particular interés e incumbencia, en el ámbito familiar, judicial, educativo, de la salud, etc. (OEA e INN, 2010: 21). 
Este documento plantea que la participación constituye un derecho que se hace efectivo básicamente como un proceso comunicativo, organizativo y de aprendizaje en el cual se reconocen tres momentos: El primero se refiere a la información, mencionando que la información es un derecho en sí mismo y una condición para que se dé la participación; por lo tanto, la fuente de información debe ser accesible, comprensible y de buena calidad, de modo que permita su apropiación por parte de los niños, niñas y adolescentes. El segundo es el de construcción y reconocimiento de la opinión propia, se pone énfasis en que todas las opiniones tanto individuales como colectivas se construyen a través de la reflexión, la discusión, el intercambio, la síntesis y la re-problematización.

Se dice que sin el reconocimiento de la voz propia (individual) no puede haber comunicación y por lo tanto no puede hablarse de participación. El tercer y último momento es el de expresión y escucha, señalando que la libertad de expresión requiere de instrumentos y manejo del lenguaje. Además, se reconoce que para que la expresión se concrete es necesario que exista una escucha, la cual debe ser respetuosa y receptiva (OEA e INN, 2010: 10-11).

Nos interesa señalar que la participación no puede considerarse como tal si las opiniones emitidas por las niñas, los niños y los adolescentes no influyen en las decisiones tomadas, así como en las acciones de las cuales se responsabilizan. Lo anterior implica crear las condiciones para que exista un clima en donde los miembros de la comunidad tienen la posibilidad de emitir opiniones, y observar que esas opiniones son tomadas en cuenta.

En coincidencia con Santos (2003), consideramos que la participación es la base de la convivencia y al mismo tiempo elemento indispensable para la formación de la ciudadanía, puesto que la participación posiciona a las niñas, niños y adolescentes como sujetos sociales con capacidad para expresar opiniones y decidir sobre asuntos que sean de su interés (Van Dijk et al. 2006). A decir de Zambrano (2001), en virtud de que a los actores sociales corresponde decidir y proponer qué aspectos de la vida social desean cambiar, los esfuerzos cotidianos que se llevan a cabo en el ámbito local y de grupos pequeños, como la familia, el barrio o las instituciones educativas son fundamentales en todo proceso de transformación social.

2. 


\section{IMPORTANCIA DE LA PARTICIPACIÓN DE LAS NIÑAS, NIÑOS Y ADOLESCENTES}

Para que la Participación Infantil sea una realidad, se debe promover el papel activo de las niñas y los niños en un ambiente de respeto y confianza.

La investigación al respecto de la participación infantil en México es escasa. Las experiencias reportadas en su mayoría hacen referencia al ámbito comunitario. Destacamos entre las investigaciones reportadas en el ámbito escolar las de Fierro y Fortul (2011), Zanabria, et al. (2007), Ochoa, et al. (2014), Hernández (2014) y Martínez (2014) que constituyen valiosos aportes para este campo. Específicamente, Ochoa (2015) realizó un estudio exploratorio descriptivo para indagar las ideas sobre participación que tienen los niños, niñas y adolescentes. Dentro de los resultados relevantes se puede mencionar que la idea sobre participación que muestran los niños, niñas y adolescentes es reducida, ya que ésta se limita a la emisión de una opinión, asimismo se destaca la escuela como el ámbito de participación que mayormente reconocen.

Recientemente se realizaron estudios que demuestran que el impulso a la participación de las niñas, niños y adolescentes mejora la convivencia, promueve valores democráticos y fortalece el sentido de pertenencia a la comunidad (Castro, 2017; Morales, 2017 y Pérez y Ochoa, 2017). La estrategia común para el impulso de la participación que se utilizó en los trabajos mencionados es la denominada Aprendizaje-Servicio.

La metodología de Proyectos de Aprendizaje Servicio está basada en las ideas de la escuela activa dado que la acción se propone como condición del aprendizaje, y la experiencia como el mecanismo que permite a los alumnos reflexionar y construir conocimientos. Esta metodología, además, la visualizamos como una estrategia expansiva debido a que, si bien los objetivos pueden centrarse en ciertos aprendizajes, uno de sus fines es la formación ciudadana, una formación que dote a los individuos de capacidades y actitudes para ejercer, promover y respaldar sus derechos, pero cumpliendo con las obligaciones que éstos conllevan, así como poner en práctica valores que permitan una vida más justa con los semejantes y con el medio ambiente.

De acuerdo a Tapia (2008), que cita los trabajos de Shapiro (1978), Titlebaum, P. et al. (2004) y Levine (2006), el primer caso que se documentó de un proyecto de APS sucedió en los Estados Unidos, alrededor de 1915, en el currículo de las Appalachian Folk Schools, en donde se integraron contenidos de aprendizaje, actividades de trabajo y servicio social con una intención pedagógica. 


\section{MARCO TEÓRICO}

De acuerdo a Puig y Palos (2006) no es posible encontrar una definición del aprendizaje servicio (APS) que aglutine las diferentes teorías, propósitos, tendencias y objetivos que a lo largo de la historia de su evolución ha tenido dicho concepto. Sin embargo, a decir de Tapia (2010), desde un punto de vista general, el aprendizaje servicio son experiencias o programas específicos desarrollados por un grupo de niños, niñas o jóvenes en el contexto de educación formal o informal, siendo una propuesta pedagógica y una manera de intervenir en lo social.

Se puede definir el APS como un proyecto protagonizado por los alumnos, el cual tiene como objetivo atender una necesidad de la comunidad al mismo tiempo que se planifican y mejoran los aprendizajes de los estudiantes (Tapia, 2005).

El APS se compone de dos elementos que tienen íntima relación: el aprendizaje y el servicio comunitario (Puig, 2014), según lo cual, el APS no es solo una estrategia que permite adquirir más y mejores aprendizajes; tampoco son solamente tareas de voluntariado realizadas por los alumnos para sensibilizarlos con respecto de su realidad. EI APS fomenta el aprendizaje de los estudiantes mediante la participación activa en experiencias realizadas en la comunidad, por medio de un proyecto estructurado e intencionado (Puig, et al. 2007). Así, la metodología permite que el estudiante se implique con los destinatarios del servicio que realiza, reconociendo sus necesidades y la realidad en la que viven, la cual no sería posible de conocer totalmente en las actividades llevadas a cabo en el aula (Folgueiras y Luna, 2010). Por ello, el APS se convierte en una estrategia que une el aprendizaje con el servicio con objetivos bien delimitados, con actividades estructuradas y con una intención pedagógica orientada a la formación ciudadana (Puig, 2009), y en nuestro caso énfasis en la mejora de la convivencia.

La consideración de los dos elementos antes señalados, el aprendizaje y el servicio, han servido como base para la propuesta que Tapia (2010) recupera del Service-Learning 2000 Center de la Universidad de Stanford, con la intención de profundizar las diferencias entre las actividades educativas que pueden desarrollarse en el contexto comunitario. 


\section{FIGURA 1}

Cuadrantes del aprendizaje servicio (Service-Learning 2000 Center, Service learning Quadransts, Palo Alto, CA, 1996, adaptado por Puig et al 2007)

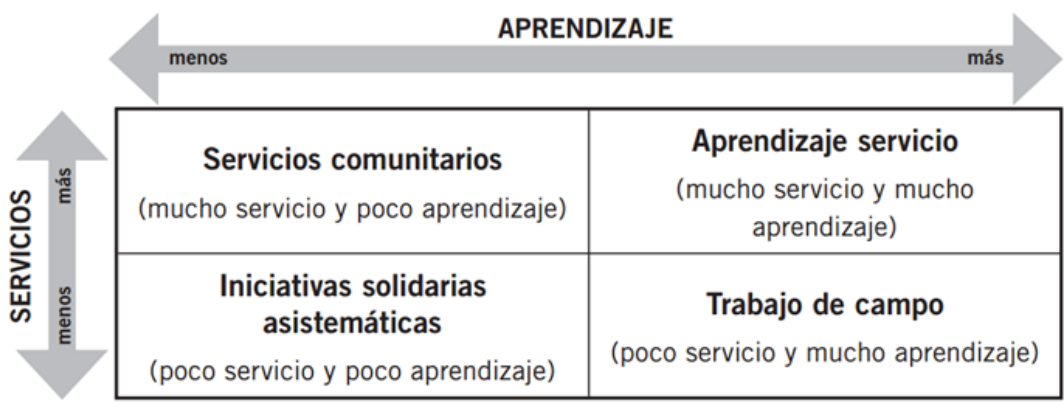

Como puede apreciarse en la figura 1 , el eje vertical señala la menor o mayor calidad del servicio solidario que se ofrece a la comunidad, en cambio el eje horizontal indica la mayor o menor integración del servicio al aprendizaje que se promueve.

En función de los ejes señalados, se pueden observar cuatro cuadrantes, los cuales permiten ver cuatro diferentes tipos de experiencias educativas en la aplicación de un proyecto de APS:

- Trabajo de campo: Se llevan a cabo acciones de investigación y práctica de los alumnos con la comunidad considerándola como objeto de estudio. Se prioriza el aprendizaje por encima del servicio. La única pretensión es lograr aprendizajes experienciales en los alumnos sin importar el servicio que se puede llegar a prestar.

- Iniciativas solidarias asistemáticas: Son actividades ocasionales y gestionadas aisladamente. El servicio está mal organizado y no hay oportunidades de aprender y reflexionar sobre lo actuado.

- Servicios comunitarios: Se gestionan a partir de una institución con un fuerte compromiso en lo social. El servicio se prioriza por encima del aprendizaje.

- Aprendizaje servicio: Son programas que atienden con calidad el servicio, pero al mismo tiempo se atienden los objetivos del aprendizaje. El servicio y el aprendizaje quedan priorizados. 
De acuerdo a Puig y Palos (2006) el APS presenta seis características deseables que deberían encontrarse en los proyectos, los cuales son:

1. El APS es un método apropiado para la educación formal y no formal, válido para todas las edades y aplicable en distintos espacios temporales.

2. EI APS se propone llevar a cabo un servicio auténtico a la comunidad que permita aprender y colaborar en un marco de reciprocidad.

3. EI APS desencadena procesos sistemáticos y ocasionales de adquisición de conocimientos y competencias para la vida.

4. EI APS supone una pedagogía de la experiencia y la reflexión.

5. EI APS requiere una red de alianzas entre las instituciones educativas y las entidades sociales que facilitan servicios a la comunidad.

6. EI APS provoca efectos en el desarrollo personal, cambios en las instituciones educativas y sociales que lo impulsan, y mejoras en el entorno comunitario que recibe el servicio.

En América Latina, Tapia (2010) destaca tres características de los programas de APS que distinguen a los proyectos de calidad, los cuales son:

1. El protagonismo activo: Implica la participación de los niños, niñas y adolescentes en el proyecto, desde su diagnóstico, elaboración, ejecución y cierre. En este sentido, darles protagonismo es permitir que sean ellos mismos los que gestionen las actividades.

2. El servicio solidario: Referido a la atención de necesidades reales de la comunidad en la que se asienta la escuela y de la que forman parte los niños, niñas y adolescentes. Implica realizar actividades con sentido social, permitiendo a los estudiantes llevar a cabo actividades concretas y adecuadas a su edad con la finalidad de solucionar problemáticas comunitarias.

3. Aprendizajes intencionalmente planificados articulados con la acción solidaria: La puesta en marcha del proyecto permite vincular los saberes y conocimientos curriculares con el servicio solidario, lo que permite la adquisición de competencias, habilidades, saberes, en contextos de aplicación real. 


\section{ETAPAS DE LOS PROYECTOS DE APS}

De acuerdo a Tapia (2010) los proyectos sociales presentan tres grandes etapas para su realización: el primero de acercamiento con la realidad, el diagnóstico de la problemática y el planteamiento del proyecto; el segundo que equivale a la ejecución del proyecto y por último el cierre y la evaluación del proyecto, por lo cual, para la realización de los proyectos de APS debería contarse con esas categorías generales.

En este sentido, Puig y Palos (2006), señalan que el desarrollo de un proyecto de APS requiere de un seguimiento de etapas bien delimitadas para conseguir los objetivos del proyecto. A partir de la revisión bibliográfica se encontró que las etapas, guías e itinerarios propuestos para llevar a cabo un proyecto de APS son múltiples y flexibles. De entre ellas destacan las propuestas del el Ministerio de Educación de la República Argentina (Programa Nacional de Educación Solidaria, 2015), el desarrollado por el Centre Promotor d'Aprenentatge Servei en Barcelona (Puig, et al, 2011) y la del grupo CLAYSS (2009).

En función de lo anterior, las etapas en las que se dividen los proyectos de APS son:

a) Motivación.

b) Diagnostico.

c) Diseño y planificación.

d) Ejecución del proyecto.

e) Cierre.

\section{DESCRIPCIÓN DE LA EXPERIENCIA}

En la presente experiencia se reportan dos procesos paralelos: por un lado, el diseño de investigación y por otro, el avance de la aplicación de la metodología de APS en las escuelas participantes.

\subsection{Método}

Se diseñó un estudio exploratorio con el objetivo general de analizar el impacto de la metodología de proyectos de APS en la convivencia escolar, así como valorar los resultados, para que, en el siguiente ciclo escolar se extienda a otras instituciones. 


\subsection{Población}

La población es de 400 estudiantes pertenecientes a 10 grupos de nivel secundaria, de 7 escuelas distintas con el acompañamiento de 53 docentes. Todas las escuelas están ubicadas en la zona metropolitana de la Cd. de Querétaro, México.

\subsection{Instrumentos}

Los instrumentos de recolección de información son: cuestionario de participación infantil, escala de convivencia escolar y observación estructurada. Los instrumentos fueron diseñados por el Observatorio de la Convivencia Escolar de la Universidad Autónoma de Querétaro. El proyecto se está llevado a cabo de acuerdo a las fases que propone el Centro Latinoamericano de Aprendizaje y Servicio Solidario (CLAYSS).

\section{RESULTADOS}

Dado que es un proyecto en curso con dos procesos paralelos, lo que se presenta en este trabajo son las problemáticas que a nivel individual mencionaron los estudiantes y la manera en la que creen que se pueden resolver (cuestionario de participación), así como el diagnóstico participativo que realizaron los estudiantes como parte de las etapas propias del proyecto.

El proyecto arrancó en el mes de febrero de 2017 en donde se convocó a una reunión con veinte escuelas secundarias. En esa reunión participaron los directores o directoras y el orientador u orientadora de las escuelas, el objetivo de esa reunión fue que el Observatorio de la Convivencia Escolar de la Universidad Autónoma de Querétaro (OCE-UAQ) en conjunto con la Unidad de Servicios Educativos del Gobierno del Estado de Querétaro presentaran la metodología para hacerles la invitación a sumarse a un proyecto piloto para valorarla, y si es el caso, extenderla a más escuelas. Una vez que escucharon y se aclararon dudas, 11 de las 20 escuelas decidieron participar.

Cuando tuvimos los datos de las escuelas que querían participar, se agendaron reuniones para que un equipo del OCE visitara cada una de las escuelas para exponer al equipo docente la metodología y el proyecto, con el fin de que las y los participantes decidieran voluntariamente el estar o no. Cuando se terminaron las presentaciones sólo 7 de las 11 escuelas decidieron continuar. En el caso de las escuelas que no continuaron se detectaron problemáticas entre docentes o entre docentes y directivos. 
Con las 7 escuelas que participan en el proyecto se han realizado las siguientes actividades: Motivación con docentes, motivación con estudiantes, actividad de cohesión grupal y el diagnóstico participativo con estudiantes. En el caso de una de las escuelas se realizó una reunión informativa con las madres y padres de familia de los dos grupos participantes.

Cabe señalar que la detección de necesidades la han realizado los estudiantes en conjunto con los docentes de cada escuela y el equipo acompañante del OCE-UAQ. Dada la problemática social que se vive en México, es difícil que las y los estudiantes salgan de la escuela para hacer un diagnóstico comunitario, es por eso que, considerando que la escuela es una comunidad, planteamos la detección de necesidades (diagnóstico) dentro de la institución.

La primera actividad que se realizó a manera de evaluación inicial de la intervención fue la aplicación del cuestionario de participación infantil, dentro de este instrumento se realizaron preguntas abiertas, dos de las cuales tienen que ver con lo que cada estudiante percibía en relación con las problemáticas que observa en su escuela, así como la forma en que ellos y ellas creen que se podrían resolver. Como eran preguntas abiertas se realizaron categorías a partir de las respuestas mismas. Los resultados se pueden observar en las siguientes tablas.

\section{TABLA 1}

Porcentaje de frecuencia de respuestas a la pregunta "Escribe una situación que te preocupe de tu escuela"

\begin{tabular}{|l|c|}
\hline \multicolumn{1}{|c|}{ Respuestas } & Porcentaje \\
\hline Trato entre alumnos & $28 \%$ \\
\hline Instalaciones escolares & $18 \%$ \\
\hline Limpieza & $12 \%$ \\
\hline Situaciones de riesgo & $8 \%$ \\
\hline No contestó & $8 \%$ \\
\hline Actitud de los docentes & $5 \%$ \\
\hline No hay ninguna & $5 \%$ \\
\hline Contaminación & $4 \%$ \\
\hline Calificaciones/Reprobar & $4 \%$ \\
\hline Otras & $3 \%$ \\
\hline No sé & $1 \%$ \\
\hline Asistencias & $1 \%$ \\
\hline Que nos separen del grupo & $1 \%$ \\
\hline Todo & $1 \%$ \\
\hline Talleres & $1 \%$ \\
\hline Total & $100 \%$ \\
\hline
\end{tabular}


Se puede apreciar en la tabla 1 que las principales problemáticas que las y los estudiantes observan a nivel individual tienen que ver con el trato entre alumnos, las instalaciones escolares y la limpieza de las mismas. Como se observará más adelante estas mismas problemáticas se ven reflejadas a nivel grupal.

La siguiente pregunta fue acerca de cómo pensaban que se podían resolver las situaciones que les preocupan. Las respuestas se observan en la tabla 2 .

\section{TABLA 2}

Porcentaje de frecuencia de respuestas a la pregunta "¿Cómo la resolverías?"

\begin{tabular}{|l|c|}
\hline \multicolumn{1}{|c|}{ Respuestas } & Porcentaje \\
\hline Dando aviso a una autoridad & $20 \%$ \\
\hline No contesto/ no sé & $17 \%$ \\
\hline Hablando/opinando & $15 \%$ \\
\hline Otras & $8 \%$ \\
\hline Limpiando & $6 \%$ \\
\hline Poniéndonos de acuerdo (alumnos) & $6 \%$ \\
\hline Sancionando & $6 \%$ \\
\hline Ayudando & $5 \%$ \\
\hline Mejorando actitudes & $4 \%$ \\
\hline Arreglando/Haciendo & $3 \%$ \\
\hline Participando & $2 \%$ \\
\hline Cambiando la escuela & $2 \%$ \\
\hline Dejando de hacerlo & $2 \%$ \\
\hline De ninguna manera & $1 \%$ \\
\hline Poniendo carteles & $1 \%$ \\
\hline Estudiando & $1 \%$ \\
\hline Con medidas de seguridad & $1 \%$ \\
\hline Total & $100 \%$ \\
\hline
\end{tabular}

Uno de los datos relevantes de las respuestas es que las y los estudiantes refieren que lo harían fundamentalmente acudiendo a una autoridad. Dado que el APS es una estrategia que pretende educar en ciudadanía y esto implica hacerse parte de los asuntos de interés común, esta respuesta es de importancia como punto de inicio de la puesta en marcha del proyecto. Seguida de esa respuesta, la que tiene un mayor porcentaje es la relacionada a no sé/ no contesto, lo cual nos hace pensar que los estudiantes no se visualizan como agentes activos para enfrentar una problemática lo que indica el rol pasivo que les asignamos a los estudiantes en las escuelas. 
Posteriormente a la aplicación del cuestionario de participación infantil y de convivencia escolar, se realizó un diagnóstico participativo para definir el servicio que las y los estudiantes realizarían, es decir, la necesidad a atender. En la mayoría de los grupos se utilizó la herramienta denominada Cartografía Social, su finalidad es que un grupo social determinado pueda tener un acercamiento real y una representación social del espacio geográfico, socio-económico, histórico-cultural en el que habita, lo que permite reconocer las circunstancias físicas en las que se viven los sujetos (Carvajal, 2005).

\section{Necesidades a atender}

El diagnóstico se realizó en dos o tres sesiones dependiendo de la agenda de cada escuela, todas las sesiones del diagnóstico se realizaron durante el mes de marzo.

Las problemáticas detectadas fueron diversas, así como las necesidades que se atenderán. Debido a lo anterior, se clasificaron de acuerdo a la finalidad de la temática que quisieron resolver, como se puede ver a continuación:

\section{Escuela 1}

- Grupo 1. Problemáticas detectadas:

- Estructurales: bancas, los bebederos, las ventanas, paredes, bocinas, pizarrón, el piso, computadoras, pinten las paredes, laboratorios, baños, canchas.

- Dinámica escolar: reglamento escolar.

- Comunitarias: alumbrado público en la calle de la escuela, incidir en la disminución de la inseguridad y la delincuencia de la comunidad.

- Necesidad a atender: Baños. Se realizarán acciones para mejorar las condiciones de los baños haciendo el aseo y pintando los espacios.

- Grupo 2. Problemáticas detectadas:

- Estructurales: bebederos, cancha deportiva, cooperativa, baños, ventiladores, butacas, mesas de trabajo.

- Necesidad a atender: Cancha deportiva escolar. Se realizarán acciones para mejorar las condiciones de la cancha deportaiva.

\section{Escuela 2}

- Grupo 1. Problemáticas detectadas:

- Estructurales: pintura de algunos espacios, una papelería, botes de basura para reciclar,

- Desarrollo social: desperdicio de comida. 
- Necesidad a atender: Hambre. Las y los estudiantes proponen realizar acciones de recolección de alimentos para después repartirlos en casas hogar y asilos de ancianos cercanos a la institución escolar.

- Grupo 2. Problemáticas detectadas:

- Estructurales: pintura en algunos espacios, mantenimiento de mobiliario, invernadero.

- Dinámica escolar: Malos tratos entre compañeros

- Necesidad a atender: Áreas verdes en la escuela. Se proponen la mejora de las jardineras escolares y de crear espacios verdes.

\section{Escuela 3}

- Grupo 1. Problemáticas detectadas:

- Estructurales: enfermería, otra cancha deportiva, áreas verdes, pintura en algunos espacios.

- Desarrollo social: Falta de opciones de esparcimiento en su comunidad y en la institución.

- Necesidad a atender: Talleres culturales y de oficios. Se proponen la creación de talleres en la escuela que permitan a las y los estudiantes pasar el tiempo libre aprendiendo algún oficio o desarrollando una actividad cultural y social. Se propone que los mismos padres de familia sean los que dirijan los talleres en el espacio escolar.

\section{Escuela 4}

- Grupo 1. Problemáticas detectadas:

- Estructurales: aulas, baños, bebederos, paredes pintadas, basura de la parte trasera de la escuela, áreas verdes, mesas y bancas de trabajo.

- Necesidad a atender: Por definir.

- Grupo 2. Problemáticas detectadas:

- Estructurales: baños, acumulación de basura (tinacos, piedras, botellas, escombros de material), cartel de entrada de la escuela, áreas verdes, salones, sillas y mesas de trabajo, bebederos, cancha deportiva, paredes pintadas con grafiti, estanquillo de alimentos.

- Necesidad a atender: Por definir

\section{Escuela 5}

- Grupo 1. Problemáticas detectadas:

- Dinámica escolar: adicciones de los estudiantes, vandalismo en la escuela. 
- Medio ambiente comunitario: basura acumulada alrededor de la escuela.

- Necesidad a atender: Medio ambiente comunitario. Se proponen llevar a cabo una campaña de limpieza del espacio comunitario en donde está inserta la escuela.

- Grupo 2. Problemáticas detectadas:

- Estructurales: limpieza de la escuela, ventiladores, quitar los grafitis, pintar los salones, pintar las bancas.

- Medio ambiente: plantar árboles, regar la escuela.

- Necesidad a atender: Arreglo del espacio escolar. Se proponen mejorar las condiciones del salón que el grupo utiliza, para posteriormente invitar a los demás estudiantes a realizar la mejora de su aula.

\section{Escuela 6}

\section{- Grupo 1. Problemáticas detectadas:}

- Estructurales: cancha deportiva, cooperativa escolar, mejora de los baños, mejora de los laboratorios, mejora de los muebles escolares.

- Medio ambiente escolar: reforestación de la escuela (huerto escolar), riego por goteo del huerto y de las áreas verdes.

- Medio ambiente comunitario: contaminación del río de la comunidad.

- Comunitarias: seguridad en la plaza de la comunidad, protección de animales en situación de calle.

- Necesidad a atender: En esta institución se eligieron varias actividades a realizar por equipos:

a) Huerto escolar. Se proponen la creación de un huerto que sea auto-sustentable, mediante la implementación de la energía solar y del riego por goteo. Se contará con el apoyo del Colectivo Tlalli quien ha participado ya con la primera charla informativa con las y los estudiantes.

b) Campaña "por los que no hablan, protección animal". Se realizarán acciones para recaudar dinero y material para ayudar a las especies (gatos y perros) que viven en la calle de la comunidad. En este proyecto se contará con la participación de la Asociación Protectora de Animales Santiago de Querétaro AC.

c) Mantenimiento escolar. Se recogerá la basura de la escuela de manera periódica y se pondrá a la venta el material que pueda ser reciclado. Lo recaudado se pondrá a disposición de la escuela o de los equipos que lo necesiten. Se solicitó el apoyo de la Asociación de Ecología y Compromiso Empresarial ECOCE AC. 
d) Limpieza y mantenimiento de los baños. Se proponen ayudar con el aseo de los baños, hacer una campaña de concientización para el buen uso de los mismos, así como repararlos y pintarlos.

\section{Escuela 7}

- Grupo 1. Problemáticas detectadas:

- Estructurales: riesgos por falta de mantenimiento en el terreno de la escuela.

- Comunitarias: grafiti en las calles.

- Medio ambiente escolar: basura de la escuela, desperdicio de agua.

- Necesidad a atender: Mantenimiento del terreno. Se proponen realizar una campaña de limpieza y mantenimiento de la escuela mediante la creación y el uso adecuado de los espacios para contener la basura.

Como puede observarse, la actividad del diagnóstico permitió que las y los estudiantes miraran con "otros ojos" el espacio escolar, permitió que se sintieran parte de éste y, además, que se dieran cuenta de que ellos pueden hacer algo para cambiar las situaciones que afectan a todos los miembros de la comunidad escolar y, en este sentido, empezar a cambiar la idea acerca de que son otros los que deben resolvernos los problemas, como se muestra en las respuestas que proporcionaron en el cuestionario inicial.

Lo anterior, se puede ratificar a partir de la evaluación parcial que se realizó el 5 de abril del año en curso. A esta reunión de evaluación acudieron algún directivo y el o la docente que coordina el proyecto en la escuela. Se aplicó un instrumento para dar seguimiento a las actividades realizadas hasta el momento, en éste se les pedía que valoraran a partir de una escala, la utilidad de las actividades aplicadas. En términos generales las y los docentes afirmaron que las actividades han servido para que las y los estudiantes: comprendan las necesidades sociales, participen de "otra forma" y para que pongan en práctica habilidades sociales.

Por otra parte, se observa que las y los docentes empiezan a cuestionar algunas de sus prácticas pues reconocen que para poner en marcha este tipo de metodología es necesario un diálogo horizontal con los estudiantes para que se cree un clima de confianza en donde prevalezca el diálogo y el consenso, y fomentar con esto la responsabilidad de las y los estudiantes. 


\section{CONCLUSIONES}

Con los resultados obtenidos hasta el momento podemos afirmar que la metodología de proyectos de Aprendizaje- Servicio en el contexto mexicano y particularmente en la $\mathrm{Cd}$. de Querétaro es pertinente para modificar las prácticas pedagógicas en los docentes, mejorar la participación de las y los estudiantes e incidir en la capacidad de iniciativa y de responsabilidad social y ciudadana de las y los participantes, elementos básicos que posibilitan la mejora de la convivencia en las instituciones escolares.

Este trabajo muestra también la importancia de la vinculación de las Instituciones de Educación Superior, con las de Educación Básica, pues a partir de este acompañamiento los docentes de educación básica no sólo aplican la metodología, sino que reflexionan sobre su práctica y sobre algunos conocimientos que, si bien los saben teóricamente, no habían encontrado la manera de articularlos con la práctica. Esto implica cambiar la mirada de los docentes como profesionales de la educación tanto de las personas externas a la institución como de los docentes mismos.

Así mismo, la puesta en marcha de este tipo de estrategias permite abonar a la pedagogía social, pues

[...] más que dirigir sus miradas hacia contenidos o saberes disciplinares fijan su atención en la dimensión social, cultural, política, cívica, etc., de quién y con quién actúan, dónde, por qué y para qué lo hacen; es decir, de los contextos y de quienes los protagonizan como sujetos o agentes de una determinada práctica educativa... (Caride; Gradaílle y Caballo, 2015, p. 6)

\section{BIBLIOGRAFÍA}

Claride, J.; Gradaílle, R. y Caballo, M. (2015). De la pedagogía social como educación, a la educación social como Pedagogía. Perfiles Educativos, vol. XXXVII, número 148 , suplemento 2015, pp. 4-10.

Bolívar, A. (2016). Educar democráticamente para una ciudadanía activa. Revista Internacional para Justicia Social, 5(1), pp. 69-87

Carvajal, A. (2005). Planeación participativa: Diagnostico, plan de desarrollo y evaluación de proyectos. Cali, Colombia: Facultad de Humanidades.

Castro, J. (2017). Formación de valores para la convivencia democrática mediante el desarrollo de la participación infantil: Un proyecto de aprendizaje basado en el servicio en una escuela de educación primaria. Tesis para obtener el grado de Maestro en Educación para la Ciudadanía. Universidad Autónoma de Querétaro, México. 
Clayss (2009). Creer para Ver. Manual para docentes y estudiantes solidarios. Buenos Aires. Fierro, C. y Fortul, B. (2011). Escuelas que construyen contextos para el aprendizaje y la convivencia democrática", en Memoria electrónica del XI Congreso Nacional de Investigación educativa. México: UNAM /COMIE.

Folgueiras, P. y Luna, E. (2010). El aprendizaje y servicio, una metodología participativa que fomenta los aprendizajes. Barcelona: Universitat de Barcelona.

Hernández, M. (2014). Des-encuentros de la participación infantil en el ámbito escolar. Un acercamiento a las ideas de los docentes de educación primaria de escuelas públicas de la ciudad de Querétaro. Tesis para obtener el grado de Maestro en Educación para la Ciudadanía. Facultad de Psicología, Universidad Autónoma de Querétaro.

Martínez, I. (2014). Miradas para la participación de niños y niñas en el contexto escolar mexicano. Tesis para obtener el grado de Maestro en Educación para la Ciudadanía. Facultad de Psicología, Universidad Autónoma de Querétaro.

Morales, L. (2017). Sentido de comunidad y participación de las y los adolescentes: una experiencia de aprendizaje-servicio en el bachillerato. Tesis para obtener el grado de Maestra en Educación para la Ciudadanía. Universidad Autónoma de Querétaro, México.

OEA e INN (2010). Menú de indicadores y Sistema de Monitoreo del Derecho a la Participación de Niños, Niñas y Adolescentes. Uruguay: OEA-IIN. Disponible en: http://www.iin. oea.org/IIN2011/documentos/Menu_Indicadores_y_sistema_monitoreo.pdf

Ochoa, A. y Diez-Martínez, E. (2012). Las interacciones escolares como contexto de construcción de la convivencia en la escuela, en Gomes, C.; Ferreira, G. y Ferreira, S. (organizadores) Culturas de violencia, culturas de paz. De la reflexión a la acción de educadores y defensores de los derechos humanos. Curitiba: CRV.

Ochoa, A.; Salinas, J. y Diez-Martínez, E. (2014). La participación de niñas, niños y adolescentes condición indispensable para la construcción de la convivencia en escuelas. Libro de Actas, XIII Congreso Internacional de Formación del Profesorado, Investigar para acompañar el cambio Educativo y Social: el papel de la Universidad. Universidad de Cantabria.

Ochoa, A. (2015). Concepciones sobre participación de niñas, niños y adolescentes: Su importancia en la construcción de la convivencia escolar, en Cultura, Educación y Sociedad 6(2). Disponible en: http://revistascientificas.cuc.edu.co/index.php/ culturaeducacionysociedad/article/view/840

Pérez, L. y Ochoa, A. (2017). La participación de los estudiantes en una escuela secundaria: retos y posibilidades para la formación ciudadana. Revista Mexicana de Investigación Educativa, 72, vol. 22, pp.176-207. Disponible en: http://www.comie.org.mx/v1/ revista/portal.php?idm $=$ es $\&$ sec $=S C 03 \& \&$ sub $=S B B \& c r i t e r i 0=A R T 72008$

Programa Nacional de Educación Solidaria (2015). Itinerario y Herramientas para desarrollar un proyecto de Aprendizaje-Servicio. Sergio Rial (Coord.). Ciudad Autónoma de Buenos Aires: Ministerio de Educación de la Nación.

Puig, J. M. (2014). En busca de otra forma de vida. En revista digital de la Asociación Convives, vol. 7, 32-37 Madrid.

Puig, J. M. (coord.) (2009). Aprendizaje servicio (ApS). Educación y compromiso cívico. Barcelona: Graó.

Puig, J. M., Martín, X. y Batlle, R. (2011). Cómo iniciar un proyecto de aprendizaje y servicio solidario. Bilbao: Fundazioa Zerbikas. 
Puig, J. M., Battle, R., Carme, B., Palos, J. (2007). Aprendizaje servicio. Educar para la ciudadanía. Barcelona: Octaedro-Ministerio de Educación y Ciencia-Centro de Investigación y Documentación Educativa.

Puig, J. M., y Palos, J. (2006). Rasgos pedagógicos del Aprendizaje y Servicio. Cuadernos de pedagogía, nº 357, 60-63.

Santos, M. (2003). Participar es aprender a convivir. En Aprender a convivir en la escuela. Madrid: Akal.

Tapia, M. N. (2005). La pràctica solidària coma a pedagogia de la ciutadania activa. Barcelona: Fundació Jaume Bofill y Universitat Oberta de Catalunya.

Tapia, M. N. (2008). Aprendizaje-servicio en la educación superior: una mirada analítica desde los protagonistas. Buenos Aires: Ministerio de Educación Presidencia de la Nación.

Tapia, M. N. (2010). La propuesta pedagógica del "aprendizaje-servicio": una perspectiva Latinoamericana. Revista científica TzhoeCoen. Chiclayo, pp. 23-44.

UNESCO (1993). Discurso del Director General de la UNESCO. Congreso Internacional sobre Educación, Derechos Humanos y Democracia. Montreal. Human Rights Teaching, núm. 8, pp. 26-33.

Van Dijk, S., Menéndez, M. J. y Gómez, A. (2006). Participación Infantil, un marco para pensar la noción de la formación. México: Save the Children. Disponible en: http://www. savethechildrenmexico.org.mx/website2008/pdf/Part \%20Infantil.pdf

Zanabria, M., Fragoso, B. y Martínez, A. (2007). Experiencias de participación infantil en Tlaxcala y Ciudad de México. Tramas, 28, pp. 121-140.

Zambrano, M. (2001). ¿Por qué promover la participación?, en Corona, Y. y Morfín,M. Diálogos de saberes sobre participación infantil. México, DF: UAM. 
